\title{
Factors Influencing Xanthine Oxidase Activity in Rat Skin
}

\author{
Walter D. Block and Doris V. Johnson \\ From the Department of Dermatology and Syphilology and the Institute of \\ Industrial Health, Medical School, University of Michigan, Ann \\ Arbor, Michigan \\ Received October 8, 1954
}

\section{INTRODUCTION}

One of the earliest studies reporting the presence of xanthine oxidase in rat skin was made by Barry et al. (1). Westerfeld and Richert (2), in a study of the distribution of this enzyme in the tissues of the white rat, confirmed this finding and reported that activity in the skin accounted for approximately $20 \%$ of the total xanthine oxidase of the rat. These authors did not study the enzyme in detail in this tissue because of the difficulty in preparing skin homogenates.

Evidence has been accumulated which would seem to indicate that a purine-catabolizing system exists in rat skin $(3,4)$. The present work represents a study of xanthine oxidase in skin, designed to provide more detailed information concerning its significance in skin metabolism. The specific purposes of this investigation were: $(a)$ to attempt to demonstrate changes in skin xanthine oxidase activity with the age of the rat, and (b) to compare xanthine oxidase in skin and liver.

\section{Methods}

White rats of the Wistar strain, ranging in age from 7 to 90 days, were used in these studies. Rats were weaned at 21 days unless otherwise noted, and were fed a standard Rockland rat diet. The animals were sacrificed by decapitation, and the hair was removed from the back and abdomen as completely as possible by clipping and dry shaving. During the removal of the shaved skin from the carcass, the subcutaneous fat and underlying tissue were simultaneously removed. Histological examination of this type of preparation showed it to consist almost entirely of dermis and epidermis; no muscle tissue, and only traces of subcutaneous fat remained.

The skin was then cut into small pieces. Three grams of tissue was extracted 
with $10 \mathrm{ml}$. of cold $0.04 M$ phosphate buffer ( $\mathrm{pH} 7.45$ ) by grinding with sand in a cold mortar and pestle for $15 \mathrm{~min}$. The material was then centrifuged at $0^{\circ} \mathrm{C}$. and 3000 r.p.m. for 5 min. The supernatant fluid was used as the active enzyme preparation. The xanthine oxidase activity of these extracts was determined in the Warburg apparatus by two procedures: the Axelrod-Elvehjem technique (5) and the Westerfeld-Richert technique (6).

One milliliter of enzyme preparation was incubated with $0.15 \mathrm{ml}$. of $0.05 M$ hypoxanthine dissolved in $0.05 \mathrm{~N} \mathrm{NaOH}$. The flasks were gassed with oxygen for 10 min. After a preliminary incubation period (10 min. for skin, $40 \mathrm{~min}$. for liver) readings were taken every $15 \mathrm{~min}$. Activities were based on oxygen consumption during the first hour. Enzyme activity is expressed in xanthine oxidase units, that is, in microliters oxygen/g. dry weight/hr. Each value shown in the tables represents the average of several delerminations. In the experiments involving very young rats, skins were pooled from litter mates of approximately equal weight to provide enough experimental material.

\section{Results and Discussion}

The difficulties involved in obtaining tissue preparations represent an important limiting factor in the study of skin metabolism. Consequently, a preliminary objective in this study was the development of a technique for obtaining active and consistent xanthine oxidase preparations from rat skin. Using the extraction technique described, assays for skin xanthine oxidase activity on a large number of adult rats gave reasonably consistent values, ranging from 195 to 230 units by the AxelrodElvehjem procedure, and from 210 to 260 units by the WesterfeldRichert procedurc. These values compare favorably with values for xanthine oxidase activity in rat skin reported by Westerfeld (2).

\section{Variations in Skin Xanthine Oxidase Activity with Age}

In a study concerning skin metabolism, Barron et al. (7) reported that the respiration of young rat skin is considerably higher than that of the adult rat. At 6 days, Barron found a $Q_{\mathrm{O}_{2}}$ value of 3.57. At 41 days, the $Q_{\mathrm{O}_{2}}$ had dropped to 0.81 , remaining at essentially this value throughout the remaining time interval studied (516 days). An attempt was therefore made to determine whether xanthine oxidase activity in skin also shows marked variations with age. Results of such a study are recorded in Table $I$. The decrease in endogenous $Q_{\mathrm{O}_{2}}$ values with increasing age is demonstrated. The $Q_{\mathrm{O}_{2}}$ values shown in Table $\mathrm{I}$ are considerably lower than Barron's figures. This difference may be attributed to the fact that the $Q_{O_{2}}$ values reported here were determined on a skin extract, while Barron's (7) work was done with skin slices, and therefore represents more nearly physiological conditions. 


\section{TABLE I}

Endogenous $Q_{\mathrm{O}_{2}}$ and Xanthine Oxidase Values of Skin Extracts from Rats of Various Ages, Weaned at 21 Days

\begin{tabular}{c|c|c|c}
\hline \multirow{2}{*}{ Age } & \multicolumn{3}{|c}{ Xanthine oxidase activity } \\
\cline { 2 - 4 } & Endogenous $Q_{02}$ & Axelrod-Elvehjem & Westerfeld-Richert \\
\hline days & cu. mm. $O_{2} / m g \cdot / h r$. & $c u . m m . O_{2} / \mathrm{g} . / \mathrm{hr}$. & cu. mm. $O_{2} / \mathrm{g} \cdot / \mathrm{hr}$. \\
7 & 0.22 & 26 & 95 \\
14 & 0.14 & 23 & 128 \\
21 & 0.10 & 40 & 105 \\
28 & 0.05 & 207 & 223 \\
36 & 0.04 & 227 & 273 \\
45 & 0.03 & 230 & 270 \\
90 & 0.03 & 215 & 235 \\
\hline
\end{tabular}

Further consideration of the data recorded in Table I shows that xanthine oxidase activity in the skin did not change significantly during the first 21 days after birth. During the 7-day period immediately following weaning (21-28 days), xanthine oxidase activity rose sharply. Beyond this period there was essentially no change in activity.

Striking differences in the values for xanthine oxidase activity of young rats (up to 21 days) were obtained using the two procedures described. The procedure of Westerfeld and Richert involves the addition of methylene blue to the flasks in which endogenous respiration is determined. The function of methylene blue is assumed to be elimination of endogenous xanthine oxidase activity by removal of substrate during the preliminary period: Since skin has a very low endogenous respiration, differences between the two methods should not be great in skin. These differences are, in fact, most marked during the period from 7 to 21 days, when the endogenous respiration is highest. The WesterfeldRichert procedure is based on the assumption that methylene blue affects only purine substrates. If methylene blue affects substrates other than purines, this procedure would give false high values.

Although the change in activity during the period from 21 to 28 days is much greater with the Axelrod-Elvehjem technique, the same qualitative relationship between age and xanthine oxidase activity exists, using either method.

The question arose as to whether the change in activity noted during the period from 21 to 28 days resulted from the change from a suckling 
TABLE II

Skin Xanthine Oxidase Activity in Rats Weaned at 88 Days

\begin{tabular}{c|c|c}
\hline \multirow{2}{*}{ Age } & \multicolumn{2}{|c}{ Xanthine oxidase activity } \\
\cline { 2 - 3 } & Axelrod-Elvehjem & Westerfeld-Richert \\
\hline days & cu. mm. $0_{2 / g . / h r .}$ & cu. mm. O2/g./hr. \\
21 & 40 & 105 \\
28 & 80 & 139 \\
36 & 212 & 242 \\
45 & 232 & 256 \\
\hline
\end{tabular}

diet to a stock diet, or to an effect of age independent of diet. Another series of rats was studied in which the period before weaning was increased from 21 to 28 days. Results of this study are summarized in Table II. Skin xanthine oxidase activity in these rats is slightly higher at 28 days than the values obtained at 21 days. It is still considerably lower, however, than activities resulting after 7 days on the stock diet. In this group of rats, activities again reached a maximal value during the first 7 days on a stock diet.

Liver Xanthine Oxidase Activity in Rats of Various Ages

Westerfeld and Richert (2) have studied the distribution of xanthine oxidase in tissues of the white rat. Liver had the highest activity, accounting for approximately $67 \%$ of the total activity, and averaging

TABLE III

Liver Xanthine Oxidase Activity in Rats of Various Ages, Weaned at 21 Days

\begin{tabular}{c|c|c}
\hline \multirow{2}{*}{ Age } & \multicolumn{2}{|c}{ Xanthine oxidase activity } \\
\cline { 2 - 3 } & Axelrod-Elvehjem & Westerfeld-Richert \\
\cline { 2 - 3 } days & cu. mm. $O_{2} / g . / h r$. & $c u . m m . O_{2} / g . / h r$. \\
7 & 0 & 180 \\
14 & 0 & 440 \\
21 & 0 & 380 \\
28 & 60 & 708 \\
36 & 223 & 583 \\
45 & 505 & 1150 \\
90 & 1100 & 1750 \\
\hline
\end{tabular}


$1862 \mathrm{cu} . \mathrm{mm} . \mathrm{O}_{2} /$ g. dry wt./hr. Skin was reported to be the second most active tissue with respect to total xanthine oxidase activity. In view of the importance of liver as a site of xanthine oxidase activity, a comparison of skin and liver xanthine oxidase activity was made.

The values for liver xanthine oxidase activity are shown in Table III. The results are by no means so clearly defined as the results for skin activity. There is a rather wide variability even between rats of the same age group. During the period from 7 to 21 days, there was no evidence of liver xanthine oxidase activity by the Axelrod-Elvehjem method. By the procedure of Westerfeld and Richert, the values ranged from 180 units at 7 days to 380 units at 21 days. Beyond the age of 28 days there was a gradual increase in activity throughout the period studied (90 days). No marked increase immediately following weaning is evident.

\section{Effect of Inanition on Skin and Liver Xanthine Oxidase Activity}

Inanition has been shown to produce marked decreases in liver xanthine oxidase activity (8). It was of interest, therefore, to determine whether skin xanthine oxidase activity is also influenced by inanition. Adult rats, fasted $72 \mathrm{hr}$., showed expected decreases in liver xanthine oxidase activity. The fasting animals showed activities ranging from 140 to 630 units, the group of control animals showing activities ranging from 890 to 1270 units. Skin xanthine oxidase, on the other hand, was unchanged during the period of fasting, activities averaging 270 units for both experimental and control animals. These experiments would seem to indicate that skin xanthine oxidase, once it has reached its maximal level of activity, is relatively resistant to inanition.

\section{SUMMary}

1. A method is described for preparing skin extracts which show reasonably consistent xanthine oxidase activities.

2. Levels of xanthine oxidase in skin do not change significantly during the first 21 days after birth. A marked increase in skin xanthine oxidase activity occurs during the period from 21 to 28 days, corresponding to the first 7 days on a stock diet. The effect of the change in diet on liver xanthine oxidase activity was less marked, results indicating a gradual increase in activity with increasing age.

3. Fasting for $72 \mathrm{hr}$. did not affect the level of xanthine oxidase in rat skin extracts. Liver xanthine oxidase was markedly depleted. 


\section{REFERENCES}

1. Barry, G., Bunbury, E., and Kennaway, E. L., Biochem. J. 22, 1102 (1928).

2. Wosterfecid, W. W., And Richent, D. A., Proc. Soc. Exptl. Biol. Med. 71, 181 (1949).

3. Johnson, D. V., ANd Block, W. D., Federation Proc. 13, 237 (1954).

4. Block, W. D., AND Johnson, D. V., J. Invest. Dermatol. 23, 471 (1954).

5. Axelrod, A. E., ANd Elvehjem, C. A., J. Biol. Chem. 140, 725 (1941).

6. Westerfeeld, W. W., and Richert, D. A., J. Biol. Chem. 199, 393 (1952).

7. Barron, E. S. G., Meyer, J., And Miller, Z. B., J. Invest. Dermatol. 11, 97 (1948).

8. Miller, L. L., J. Biol. Chem. 172, 113 (1948). 